\title{
Effects of a concurrent musical task on a unimanual skill
}

\author{
WILLIAM J. THOMSON and SUSANNE CLAUSNITZER \\ University of Michigan, Dearborn, Michigan 48128
}

\begin{abstract}
In contrast to general findings of interference between concurrent tasks, Kinsbourne and Cook (1971) found evidence of facilitation of a right-hemisphere mediated motor task by simultaneous verbalization. The present study utilized 16 human subjects and found comparable facilitation of a left-hemisphere mediated motor task by a concurrent musical task. The results were interpreted as supporting Kinsbourne and Cook's model of hemispheric competition and facilitation.
\end{abstract}

Several studies have indicated that human subjects will generally exhibit impaired efficiency if required to perform two tasks simultaneously (e.g., Fitts \& Posner, 1967). When considering this phenomenon in terms of hemispheric asymmetry, several investigators have found interference of right-hand functioning by various simultaneous verbal tasks (Bowers, Heilman, Stax, \& Altman, 1978; Hicks, 1975; Johnson \& Kozma, 1977; Kinsbourne \& Cook, 1971), as well as interference of left-hand performance by humming (Lomas \& Kimura, 1976) and by a visuospatial task (Bowers et al., 1978). However, Kinsbourne and Cook (1971) reported that performance of a second simultaneous task, under certain conditions, could facilitate performance. Specifically, they found that in right-handed subjects, time of balancing a dowel rod on the left index finger was increased when the subject was simultaneously required to repeat sentences. This result was interpreted in terms of a model that postulated interference between tasks when both tasks were controlled by the same cerebral hemisphere (e.g., right-hand balancing and verbalizing). They also suggested that when the concurrent tasks were mediated by different hemispheres (e.g., left-hand balancing and verbalizing), facilitation would occur due to a reduction in the usual interference between hemispheres by the distracting task. Later studies have failed to support this interpretation. Johnson and Kozma (1977) failed to replicate the facilitative effect of verbalizing on left-hand functioning and also failed to find a facilitative effect for humming on right-hand performance. Hicks (1975) also failed to find righthand facilitation with a simultaneous humming task and, in fact, found verbal and humming tasks to have comparable interfering effects.

The purpose of the present study is to provide a further test of the Kinsbourne and Cook (1971) facilitation hypothesis by using a musical task somewhat different from those utilized in previous studies.

Requests for reprints should be sent to William J. Thomson, Department of Behavioral Sciences, University of MichiganDearborn, 4901 Evergreen Road, Dearborn, Michigan 48128.

\section{METHOD}

\section{Subjects}

The subjects were eight female and eight male introductory psychology students participating in partial fulfillment of a course requirement. All subjects reported a strong right-hand preference.

\section{Apparatus}

A wooden dowel rod measuring $1.3 \times 45.7 \mathrm{~cm}$ was used. Ten musical themes were played by guitar and recorded, each beginning and ending with the first pitch of the $\mathrm{C}$ major eightnote scale, with the remaining notes randomly chosen. The first phrase had six notes and the second phrase had five notes, with the two phrases set in a slow $4 / 4$ meter. The resulting themes were designed to represent a familiar and simple musical form, while presenting tunes unknown to the subject.

\section{Procedure}

The primary task of vertically balancing the dowel rod and the secondary task of humming aloud the designated musical theme were described to the subject. The subject then practiced balancing the dowel rod on the index finger, while standing, for a total of $11 \mathrm{~min}$, sharing time equally between the hands, with 1-min trials and 1-min intermissions. The subject then proceeded to the experimental trials.

Four females and four males were randomly assigned to each of two groups. After the practice session, 20 experimental trials were given to each subject, with only the order of the specific condition differing for the two groups. The trials were counterbalanced between hands (right and left) and between the two conditions (humming and nonhumming) as follows: RN, $\mathrm{LN}, \mathrm{RH}, \mathrm{LH}$, repeated five times for one group, and $\mathrm{LH}, \mathrm{RH}$, LN, RN, for the other group. The nonhumming condition was identical in form to the practice trials. The humming condition differed only in that the subject was asked to listen to one of the musical themes and then to repeat the pitches, humming aloud continuously (without correction) while balancing the dowel rod. For each trial, the le igth of time the subject was able to balance the dowel rod was recorded. A trial was concluded if the balancing time exceeded $180 \mathrm{sec}$.

\section{RESULTS}

The mean of the median balancing times (in seconds) for each condition was: $\mathrm{LH}=5.2, \mathrm{LN}=7.7, \mathrm{RH}=9.0$, and $R N=8.1$. A Friedman analysis of variance indicated significant main effects $\left[\chi^{2}(3)=306.5, \mathrm{p}<.001\right]$. 
Wilcoxon pairwise comparisons revealed: (1) $\mathrm{LH}<\mathrm{LN}$ $(\mathrm{z}=3.51, \mathrm{p}<.001),(2) \mathrm{RN}<\mathrm{RH}(\mathrm{z}=2.69, \mathrm{p}<.01)$, (3) $\mathrm{LH}<\mathrm{RH}(\mathrm{z}=3.51, \mathrm{p}<.001)$, and (4) no significant difference between $\mathrm{LN}$ and $\mathrm{RN}(\mathrm{z}=.15)$.

\section{DISCUSSION}

In line with previous results, a performance decrement occurred during left-hand balancing when the subject was required to hum, in comparison with both the $\mathrm{LN}$ and the RH conditions. This finding may be interpreted in terms of the Kinsbourne and Cook (1971) model as due to interference between concurrent tasks controlled by the same hemisphere. In contrast to Hicks (1975) and Johnson and Kozma (1977), a facilitation of right-hand performance with a simultaneous musical task was found. One possible reason for the difference in results may be due to the type of musical material that was used in each study. In the Hicks study, segments of popular songs of the 1950s were used, and while Johnson and Kozma do not precisely describe their musical material, it is suggested that the music was composed of unfamiliar melodies of varying rhythm. Since the present study maintained a constant rhythm while varying tones, all of the results can be subsumed under the Kinsbourne and Cook model if it is assumed that rhythm is a left-hemisphere function, whereas tone is mediated by the right hemisphere, as suggested by Johnson and Kozma (1977).
If such an assumption is true, the present results may be interpreted as support for the Kinsbourne and Cook (1971) assertion that performance mediated by a particular hemisphere may be facilitated by distracting the other hemisphere and thus interfering with the usual competition between the hemispheres.

\section{REFERENCES}

Bowers, D., Heilman, K. M., Satz, P., \& Altman, A. Simultaneous performance on verbal, nonverbal and motor tasks by right-handed adults. Cortex, 1978, 14, 540-556.

Fitts, P. M., \& Posner, M. I. Human performance. Belmont, Calif: Brooks-Cole, 1967.

Hicks, R. Intrahemispheric response competition between vocal and unimanual performance in normal adult human males. Journal of Comparative and Physiological Psychology, 1975, 89, 50-60.

Johnson, O., \& Kozma, A. Effects of concurrent verbal and musical tasks on a unimanual skill. Cortex, 1977, 13, 11-16.

Kinsbourne, M., \& Cook, J. Generalized and lateralized effects of concurrent verbalization on a unimanual skill. Quarterly Journal of Experimental Psychology, 1971, 23, 341-345.

Lomas, J., \& Kimura, D. Intrahemispheric interaction between speaking and sequential manual activity. Neuropsychologia, 1976, 14, 23-24.

(Received for publication October 24, 1980.) 